\title{
CURVATURE FORMS FOR LORENTZ 2-MANIFOLDS
}

\author{
JOHN T. BURNS
}

\begin{abstract}
As a converse to the Gauss-Bonnet theorem for Lorentz metrics on 2-manifolds, we show that if $\bar{\Omega}$ is a 2 -form on the torus $T^{2}$ and $\int_{T^{2}} \bar{\Omega}=0$ then $\bar{\Omega}$ is the curvature form of some Lorentz metric on $T^{2}$.
\end{abstract}

Introduction. In this paper, we will show that if $\bar{\Omega}$ is a 2 -form on the torus $T^{2}$ and $\int_{T^{2}} \bar{\Omega}=0$, then $\bar{\Omega}$ is the curvature form of some Lorentz metric on $T^{2}$. For compact oriented 2-dimensional Riemannian manifolds, this "converse to the Gauss-Bonnet theorem" has been proved by Wallach and Warner [5], in which crucial use is made of the Hodge decomposition theorem for harmonic forms. Avez [2, Chapitre III] has extended the Hodge theorem to include certain Lorentz metrics on $T^{2}$. It is precisely this extension that enables us to extend the Wallach and Warner result to the Lorentzian case.

Definitions, notation, and preliminary results. For convenience, we recall the terminology of harmonic forms and state without proof the main results of [2], which one may consult for detailed proofs.

Let $V$ be an $n$-dimensional real vector space with a symmetric nondegenerate bilinear form $g$ having $s$ negative squares and $n-s$ positive squares when written in canonical form. Let $\hat{V}$ be the dual space of $V$. Then $g$ induces a similar bilinear form $\hat{g}$ on $\hat{V}$. A basis $\left\{X_{1}, \ldots, X_{n}\right\}$ of $V$ is called orthonormal if $g\left(X_{i}, X_{j}\right)= \pm \delta_{i j}$. Define numbers $e_{i}= \pm 1$ by $g\left(X_{i}, X_{j}\right)=e_{i} \delta_{i j}$. If $\left\{\theta_{1}, \ldots, \theta_{n}\right\}$ is the corresponding dual basis, then $\hat{g}\left(\theta_{i}, \theta_{j}\right)=e_{i} \delta_{i j} \cdot g_{\Lambda}$ also induces a similar form denoted by $g_{p}$ on each of the vector spaces $\Lambda^{p} V$, for $1 \leqslant p \leqslant n-1$. For $\lambda, \mu \in \Lambda^{p} V$, where $\lambda=\alpha_{1} \wedge \cdots \wedge \alpha_{p}$ and $\mu=\beta_{1}$ $\wedge \cdots \wedge \beta_{p}, \quad \alpha_{i}, \quad \beta_{j} \in \hat{V}, \quad 1 \leqslant i \leqslant p, \quad 1 \leqslant j \leqslant p$, define $g_{p}(\lambda, \mu)=$ $\operatorname{det}\left[\hat{g}\left(\alpha_{i}, \beta_{j}\right)\right]$. If $V$ is oriented, then for $1 \leqslant p \leqslant n-1$, there is an isomorphism $*: \Lambda^{p} \hat{V} \rightarrow \Lambda^{n-p} \hat{V}\left[4\right.$, p. 15]. Let $\Theta_{I}=\theta_{i_{1}} \wedge \cdots \wedge \wedge \theta_{i_{p}}, 1 \leqslant i_{1}<i_{2}$ $<\cdots<i_{p} \leqslant n$, be a basis element of $\Lambda^{p} \hat{V}$. Then $* \Theta_{I}=$ $(\operatorname{sgn} \sigma) g_{n-p}\left(\Theta_{J}, \Theta_{J}\right) \Theta_{J}$, where $J$ is the set of indices complementary to $I$ in $\{1,2, \ldots, n\}$, and $\sigma$ is the permutation $\{I, J\} \rightarrow\{1,2, \ldots, n\}$. It follows that $*-1: \Lambda^{n-p} \hat{V} \rightarrow \Lambda^{p} \hat{V}$ satisfies $*^{-1}=(-1)^{p(n-p)+s} *$. Note in particular, that if $n=2$ and $p=1$, then $\Lambda^{1} \hat{V}=\hat{V}$, and for the orthonormal basis $\left\{\theta_{1}, \theta_{2}\right\}$ of $V$ it follows that $*: \hat{V} \rightarrow \hat{V}$ is defined by $* \theta_{1}=g\left(\theta_{2}, \theta_{2}\right) \theta_{2}=$ $e_{2} \theta_{2}$ and $* \theta_{2}=-g\left(\theta_{1}, \theta_{1}\right) \theta_{1}=-e_{1} \theta_{1}$.

Received by the editors June 28, 1976.

AMS (MOS) subject classifications (1970). Primary 53C50. 
Now let $M$ be an oriented Lorentz manifold of dimension $n$ with metric $g$, and let $E^{p}(M)$ be the vector space of smooth $p$-forms on $M$. With the aid of the operator * and the exterior derivative $d$, define an operator $\delta: E^{p}(M) \rightarrow$ $E^{p-1}(M), 1 \leqslant p \leqslant n$, which associates to each $p$-form $\alpha$ a $(p-1)$-form $\delta \alpha=(-1)^{p_{*}-1} d * \alpha$. Finally, for each $p, 0 \leqslant p \leqslant n$, define the linear operator $\Delta: E^{p}(M) \rightarrow E^{p}(M)$ by $\Delta=d \delta+\delta d$. On $E^{0}\left(R^{2}, g\right)$, that is on $C^{\infty}$ functions defined on $R^{2}$ with the flat metric $g: d s^{2}=d x^{2}-d y^{2}, \Delta$ is the operator $(-1) \square=(-1)\left(\partial^{2} / \partial x^{2}-\partial^{2} / \partial y^{2}\right)$. Recall that the operators $*$ and $\Delta$ commute. The Hodge decomposition theorem for compact oriented Riemannian manifolds [6] states that $E^{p}(M)=d\left(E^{p-1}(M)\right) \oplus \delta\left(E^{p+1}(M)\right)$ $\oplus H^{p}(M)$, where $H^{p}(M)=\left\{\omega \in E^{p}(M) \mid \Delta \omega=0\right\}$, and the decomposition is orthogonal with respect to the scalar product $\langle\alpha, \beta\rangle=\int_{M} \alpha \wedge * \beta$ for $\alpha, \beta$ $\in E^{p}(M)$. Avez calls a compact Lorentz manifold $(M, g)$ strongly de Rham if the Hodge decomposition theorem is true on $(M, g)$.

We now consider the torus $T^{2}$ with a flat Lorentz metric $g$ defined by its components $g_{11}=a^{2}, g_{22}=-b^{2}, g_{12}=g_{21}=0$. The key theorems necessary for our result are now stated, where the second number is the original number in Avez [2].

THEOREM (A.1) (11, III). If $\left|g_{11} / g_{22}\right|=a^{2} / b^{2}$ is an irrational algebraic number, then $T^{2}$ with the metric $g$ is strongly de Rham.

TheOREM (A.2) (9, III). On a strongly de Rham manifold $M, \operatorname{dim} H^{p}(M)=$ $B_{p}(M)$, where $B_{p}(M)$ is the pth Betti number of $M$.

\section{Main result.}

THEOREM. Let $M$ be a compact connected orientable 2-dimensional manifold which admits a Lorentz metric. Let $\bar{\Omega}$ be a 2-form on $M$. Then a necessary and sufficient condition that $\bar{\Omega}$ be the curvature form of some Lorentz metric on $M$ is that $\int_{M} \bar{\Omega}=2 \pi \chi(M)$, where $\chi(M)$ is the Euler characteristic of $M$.

Proof. As is well known, the only compact connected orientable 2-dimensional manifold which admits a Lorentz metric is the 2-torus $T^{2}$. Hence the necessary and sufficient condition becomes $\int_{T^{2}} \bar{\Omega}=0$. Necessity follows from the Gauss-Bonnet theorem for indefinite metrics [1], [3]. For the sufficiency, let $g$ be a strongly de Rham Lorentz metric on $T^{2}$, which exists by Theorem (A.1), and let $\Omega$ be the curvature form for $g$. Then $\int_{T^{2}}(\Omega-\bar{\Omega})=0$. By Theorem (A.2), $\operatorname{dim} H^{0}\left(T^{2}\right)=B_{0}\left(T^{2}\right)=1$, which implies that the harmonic 0 -forms, i.e. the harmonic functions, are constants. Now let $\eta$ be a harmonic 2-form, i.e. $\Delta \eta=0$. Then since $\Delta(* \eta)=*(\Delta \eta)=0, * \eta$ is a harmonic function and, by the above, a constant. Then

$$
\langle\Omega-\bar{\Omega}, \eta\rangle=\int_{T^{2}}(\Omega-\bar{\Omega}) \wedge * \eta=(* \eta) \int_{T^{2}}(\Omega-\bar{\Omega})=0 .
$$

Since $\eta$ is arbitrary, we have that $\Omega-\bar{\Omega}$ is orthogonal to the harmonic 2-forms. By the Hodge theorem, there exists a 2 -form $\beta$ such that $\Delta \beta=\Omega-$ $\bar{\Omega}$. Let $\lambda=* \beta$. 
We will show that $\bar{\Omega}$ is the curvature form of the metric $\bar{g}=e^{2 \lambda} g$. Let $\left\{X_{1}, X_{2}\right\}$ be a local orthonormal frame field on $T^{2}$ for the metric $g$ and let $\left\{\boldsymbol{\theta}_{1}, \boldsymbol{\theta}_{2}\right\}$ be the corresponding local oriented coframe field. If we set $\bar{\theta}_{i}=e^{\lambda} \boldsymbol{\theta}_{i}$, then $\left\{\bar{\theta}_{1}, \bar{\theta}_{2}\right\}$ is a local coframe field for the metric $\bar{g}$. Now $\Omega=d \omega_{12}$, where $\omega_{12}$ is the connection form uniquely determined by the requirements $\omega_{12}=$ $-\omega_{12}, d\left(e_{1} \theta_{1}\right)=e_{2} \theta_{2} \wedge e_{1} \omega_{12}$, and $d\left(e_{2} \theta_{2}\right)=e_{1} \theta_{1} \wedge e_{2} \omega_{21}$ [7, p. 51]. We compute $\bar{\omega}_{12}$. Let $d \lambda=\lambda_{1} \theta_{1}+\lambda_{2} \theta_{2}$. Then

$$
\begin{aligned}
& d\left(e_{1} \bar{\theta}_{1}\right)=e^{\lambda}\left(-\lambda_{2} e_{1} \theta_{1}-e_{1} e_{2} \omega_{12}\right) \wedge \theta_{2} \\
& =\left(-\lambda_{2} e_{1} \theta_{1}+\lambda_{1} e_{2} \theta_{2}-e_{1} e_{2} \omega_{12}\right) \wedge e^{\lambda} \theta_{2}=\left(* d \lambda-e_{1} e_{2} \omega_{12}\right) \wedge \bar{\theta}_{2} .
\end{aligned}
$$

In the Riemannian case $e_{1}=e_{2}=1$, so $e_{1} e_{2}=1$ also, and we have

$$
d \bar{\theta}_{1}=\left(* d \lambda-\omega_{12}\right) \wedge \bar{\theta}_{2}=-\left(\omega_{12}-* d \lambda\right) \wedge \bar{\theta}_{2} .
$$

Hence $\bar{\omega}_{12}=\omega_{12}-* d \lambda$, the equation of Wallach and Warner. In the Lorentz case, $e_{1} e_{2}=-1$, so

$$
d\left(e_{1} \bar{\theta}_{1}\right)=-\left(e_{1} e_{2} \omega_{12}+e_{1} e_{2}(* d \lambda)\right) \wedge \bar{\theta}_{2}=-e_{1}\left(\omega_{12}+* d \lambda\right) \wedge e_{2} \bar{\theta}_{2} .
$$

Hence $\bar{\omega}_{12}=\omega_{12}+* d \lambda$. Now for 2 -forms, $\Delta=d \delta$, where $\delta=(-1) * d *$. Using this, we find the curvature form of the metric $\bar{g}=e^{2 \lambda} g$ is

$$
d \bar{\omega}_{12}=d \omega_{12}+d * d \lambda=d \omega_{12}-d \delta \beta=\Omega-\Delta \beta=\bar{\Omega} . \quad \text { Q.E.D. }
$$

\section{REFERENCES}

1. A. Avez, Formule de Gauss-Bonnet-Chern en métrique de signature quelconque, C. R. Acad. Sci. Paris 255 (1962), 2049-2051. MR 26 \#2993.

2. Essais de géométrie riemannienne hyperbolique globale, Ann. Inst. Fourier (Grenoble) 13 (1963), fasc. 2, 105-190. MR 29 \#205.

3. S. S. Chern, Pseudo-Riemannian geometry and the Gauss-Bonnet formula, An. Acad. Brasil Ci. 35 (1963), 17-26. MR 27 \#5196.

4. H. Flanders, Differential forms with applications to the physical sciences, Academic Press, New York, 1963. MR 28 \#3397.

5. N. Wallach and F. Warner, Curvature forms for 2-manifolds, Proc. Amer. Math. Soc. 25 (1970), 712-713. MR 41 \# 7581.

6. F. Warner, Foundations of differentiable manifolds and Lie groups, Scott, Foresman, Glenview, Ill., 1971. MR 45 \# 4312.

7. J. Wolf, Spaces of constant curvature, 2nd ed., 1972 (published by and available from the author).

Department of Mathematics, University of Colorado, Colorado Springs, Colorado 80907 